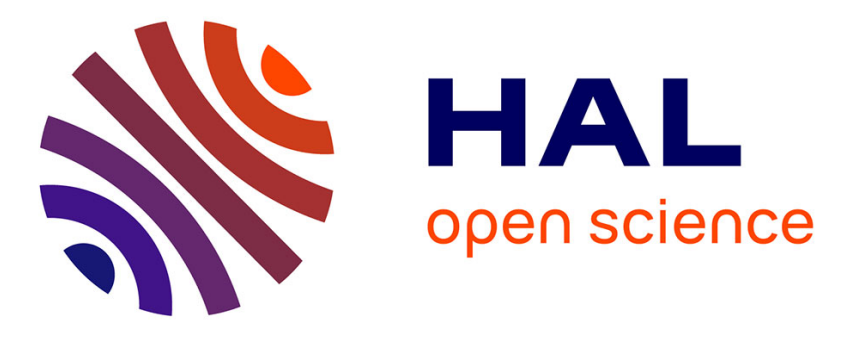

\title{
Preparation of granular Bi-2212 nanowires by electrospinning
}

Xian S Lin Zeng, Michael R Koblischka, Thomas S Karwoth, Thomas Hauet, Uwe S Hartmann

\section{> To cite this version:}

Xian S Lin Zeng, Michael R Koblischka, Thomas S Karwoth, Thomas Hauet, Uwe S Hartmann. Preparation of granular Bi-2212 nanowires by electrospinning. Superconductor Science and Technology, 2017, 30 (3), pp.035014. 10.1088/1361-6668/aa544a . hal-01569236

\section{HAL Id: hal-01569236 \\ https://hal.science/hal-01569236}

Submitted on 26 Jul 2017

HAL is a multi-disciplinary open access archive for the deposit and dissemination of scientific research documents, whether they are published or not. The documents may come from teaching and research institutions in France or abroad, or from public or private research centers.
L'archive ouverte pluridisciplinaire HAL, est destinée au dépôt et à la diffusion de documents scientifiques de niveau recherche, publiés ou non, émanant des établissements d'enseignement et de recherche français ou étrangers, des laboratoires publics ou privés. 


\title{
Preparation of granular $\mathrm{Bi}-2212$ nanowires by electrospinning
}

\author{
Xian Lin Zeng a) ${ }^{1}$ Michael R Koblischka, ${ }^{1}$ Thomas Karwoth, ${ }^{1}$ Thomas Hauet, ${ }^{2}$ and \\ Uwe Hartmann ${ }^{1}$ \\ 1) Institute of Experimental Physics, Saarland University, Campus C 63 , \\ 66123 Saarbrücken, Germany. \\ ${ }^{2)}$ Institute Jean Lamour, UMR CNRS-Université de Lorraine, \\ 54506 Vandœevre-lès-Nancy, France.
}

(Dated: 9 September 2016)

$\mathrm{Bi}_{2} \mathrm{Sr}_{2} \mathrm{CaCu}_{2} \mathrm{O}_{8}(\mathrm{Bi}-2212)$ superconducting nanowires are synthesized by the electrospinning method. Two different precursors were employed to grow the nanowires, a $\mathrm{Pb}$-doped one and an $\mathrm{Cu}, \mathrm{Ca}$-enriched one. The granular polycrystalline character was confirmed by scanning electron microscopy and X-ray diffraction measurement. The magnetic and electric properties of the samples were investigated via SQUID magnetometry and four-probe resistance measurements. The superconducting transition temperature of both systems were found to be similar (Pb-doped $84 \mathrm{~K}, \mathrm{Cu}, \mathrm{Ca}$ enriched $76 \mathrm{~K}$ ), but the transition width and the onset of irreversibility were clearly different. The magnetization loops and the resistance data demonstrate that the $\mathrm{Cu}$,Ca-enriched precursor yields samples with better superconducting properties.

a) Email: x.zeng@physik.uni-saarland.de

PACS: 74.72.-h, 74.25.Ha, 74.25.F-, 81.07.Gf

Key words: Bi-2212, high- $T_{\mathrm{c}}$, superconductor, polycrystalline, nanowire, electrospinning, doping 


\section{INTRODUCTION}

Superconducting nanowires form an attractive research field for over decades, ranging from several types of low temperature superconductors ${ }^{1}$ to high temperature cuprate superconductors $^{2}$. With decreasing size and the dimension constraint, superconducting nanowires provide magnetic and electric properties different from bulk materials, which may create some new possibilities for applications. The most famous application of superconducting nanowires are the single phonon detectors ${ }^{3,4}$. The other important aspect is the fundamental research on superconductivity in confined geometry. Theoretical work based on the BCS theory has been reported demonstrating the influence of thermal and quantum fluctuation onto the superconducting properties ${ }^{5-7}$. Furthermore, superconductivity of granular superconductors is a topic of interest ranging from bulks, thin films to one dimensional nanowires. Most of the samples investigated in the literature were produced from epitactic thin films grown by various methods and subsequently etched by ebeam lithography or focused ion beam (FIB) techniques ${ }^{8,9}$.

The sol-gel growth method is one of the popular methods producing granular, polycrystalline samples with nanometer-sized grains ${ }^{10}$. This approach can be combined with the electrospinning technique, which is a typical approach fabricating high yield, long polymer and ceramic nanowires ${ }^{11}$. After removing of the organic ingredient by the heat treatment, the nanowires are formed of single crystal-like grains chaining with each other. An important feature of these nanowires is the formation of a fabric-like, non-woven network without a substrate. Therefore, the influence of the substrate on the nanowire properties is eliminated here. The intergrain weak links supply the main contribution to the granular effect, and during the heat treatment, interconnects between the individual nanowires are formed, which are important for the current flow through the entire sample perimeter.

In this paper, we focus on the preparation of electrospun superconducting $\mathrm{Bi}_{2} \mathrm{Sr}_{2} \mathrm{CaCuO}_{8}$ (Bi-2212) nanowires from two different precursor types, (i) a Cu, Ca-enriched precursor and (ii) a Pb-doped stoichiometric precursor. In Sec. II, the procedure of the nanowire synthesis is introduced, including the morphological characterization and phase confirmation. In Sec. III, a comparison of magnetic and electric properties between $\mathrm{Pb}$ doped and undoped samples is presented, and the superconducting properties of the granular nanowire network with intergrain weak links will be investigated. Finally in Sec. IV, we will give some conclusions. 


\section{PREPARACTION AND CHARACTERIZATION}

The nanowires are fabricated by the electrospinning method and all the chemicals are commercially available from Alfa Aesar GmbH Company. For the preparation of the Bi2212 nanowire precursor, the bismuth acetate (purity of 99.999\%), strontium acetate hydrate (purity of $99.99 \%$ ), calcium acetate hydrate (purity of 99.9965\%) and copper acetate monohydrate (purity of 99.99\%) powders are weighted with the molar ratio $\mathrm{Bi}: \mathrm{Sr}: \mathrm{Ca}: \mathrm{Cu}=$ 1:1:1:2 (the sample with this precursor is named as 1112 hereafter) and dissolved in the propionic acid. The excess of calcium and copper acetates serve to suppress the impurity formation according to the reaction $\mathrm{Bi}_{2} \mathrm{Sr}_{2} \mathrm{CaCu}_{2} \mathrm{O}_{8} \rightarrow \mathrm{Bi}_{2} \mathrm{Sr}_{2} \mathrm{CuO}_{6}+\mathrm{CaCuO}_{2}$ during the thermal treatment process ${ }^{12}$. For the $5 \% \mathrm{~Pb}$-doped sample, the $\mathrm{Pb}$ acetate trihydrate (ACS purity) is added. The acetate molar ratio is adjusted to $\mathrm{Bi}: \mathrm{Pb}: \mathrm{Sr}: \mathrm{Ca}: \mathrm{Cu}=1.9: 0.1: 2: 1: 2$ (the sample with this precursor is named as $\mathrm{Pb}$-doped sample), the addition of $\mathrm{Pb}$ contributes to the formation of the Bi-2212 phase according to Refs. ${ }^{13,14}$, thus no excess of calcium and copper are applied in this case. For electrospinning, the polymer polyvinylpyrrodione (M.W. 1,300,000) is mixed to the solutions to enhance the viscosity of the precursor. All the chemicals will be completely dissolved by stirring for $15 \mathrm{~h}$ at room temperature. The electrospinning is undertaken using a MECC nanofiber electrospinner ${ }^{15}$. The parameters are shown in the table below. More details of the nanowire fabrication procedure are given elsewhere ${ }^{16,17}$.

\begin{tabular}{|l|c|}
\hline Parameter & BSCCO+PVP \\
\hline Applied voltage $(\mathrm{kV})$ & 20 \\
Fly distance $(\mathrm{mm})$ & 190 \\
Pump rate $\left(\mathrm{mL} \mathrm{h}^{-1}\right)$ & 0.1 \\
Travel speed $\left(\mathrm{mm} \mathrm{s}^{-1}\right)$ & 50 \\
Travel distance $(\mathrm{mm})$ & 100 \\
Temperature $\left({ }^{\circ} \mathrm{C}\right)$ & 22 \\
Relative humidity $(\%)$ & $30-45$ \\
\hline
\end{tabular}

TABLE I. Synthesis parameters for electrospinning of the as-prepared BSCCO fibers.

To remove the organic compounds and obtain the Bi-2212 phase, a thermal treatment is applied in a laboratory box furnace according to a differential scanning calorimetry (DSC) 
and thermogravimetric analysis (TGA) as presented in Figure 1. The temperature of the final step thermal treatment is over $800{ }^{\circ} \mathrm{C}$ as for the solid thermal synthesis of bulk materials, the sample obtains the phase at $825{ }^{\circ} \mathrm{C}$. An additional treatment at $500{ }^{\circ} \mathrm{C}$ in pure $\mathrm{O}_{2}$ environment is required to compensate the oxygen loss during the former heat treatment process.

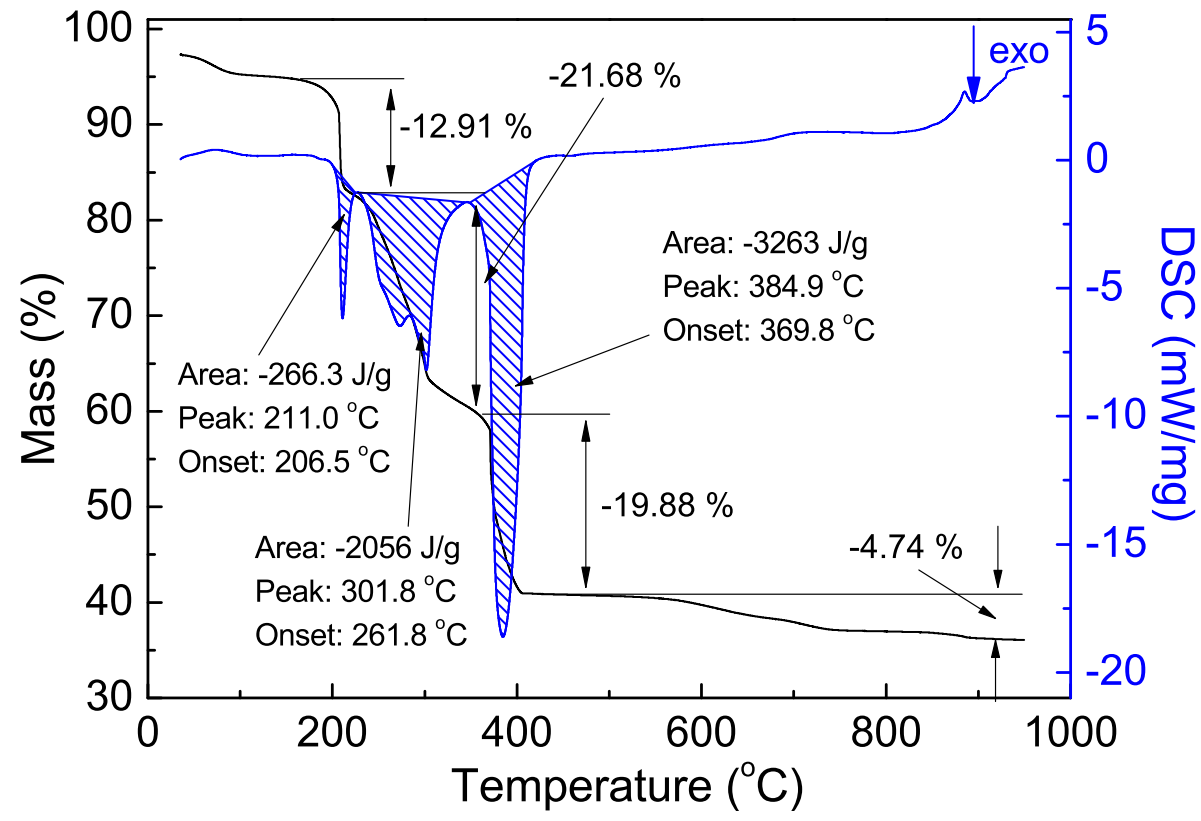

FIG. 1. Result of the differential scanning calorimetry (DSC) and thermogravimetric analysis (TGA) about the as-prepared nanowires

Figure 2 shows scanning electron microscopy (SEM) images of the nanowires at different steps of the thermal treatment, images (a) - (e) present the undoped nanowires (1112 sample), and (f) the Pb-doped one. Before the thermal treatment, the as-prepared nanowires are tenacious and straight because the polyvinylpyrrodione helps to strengthen the inner connection of the wire. As the fibers experience higher temperature treatments, the organic compound is gradually removed and the wire structure starts to bend. Meanwhile, with the crystallization process the grains grow larger. It can be seen that after the thermal treatment, the nanowires are not as smooth as the as-prepared nanowires due to the growth of the crystal grains. The junction between the grains becomes the main connection way to maintain the wire structure, instead of binding by polymer. Figure 2 (e) and (f) present the final structures of the 1112 sample and $\mathrm{Pb}$-doped sample after the $800{ }^{\circ} \mathrm{C}$ treatment and $500{ }^{\circ} \mathrm{C} \mathrm{O}_{2}$ treatment. 

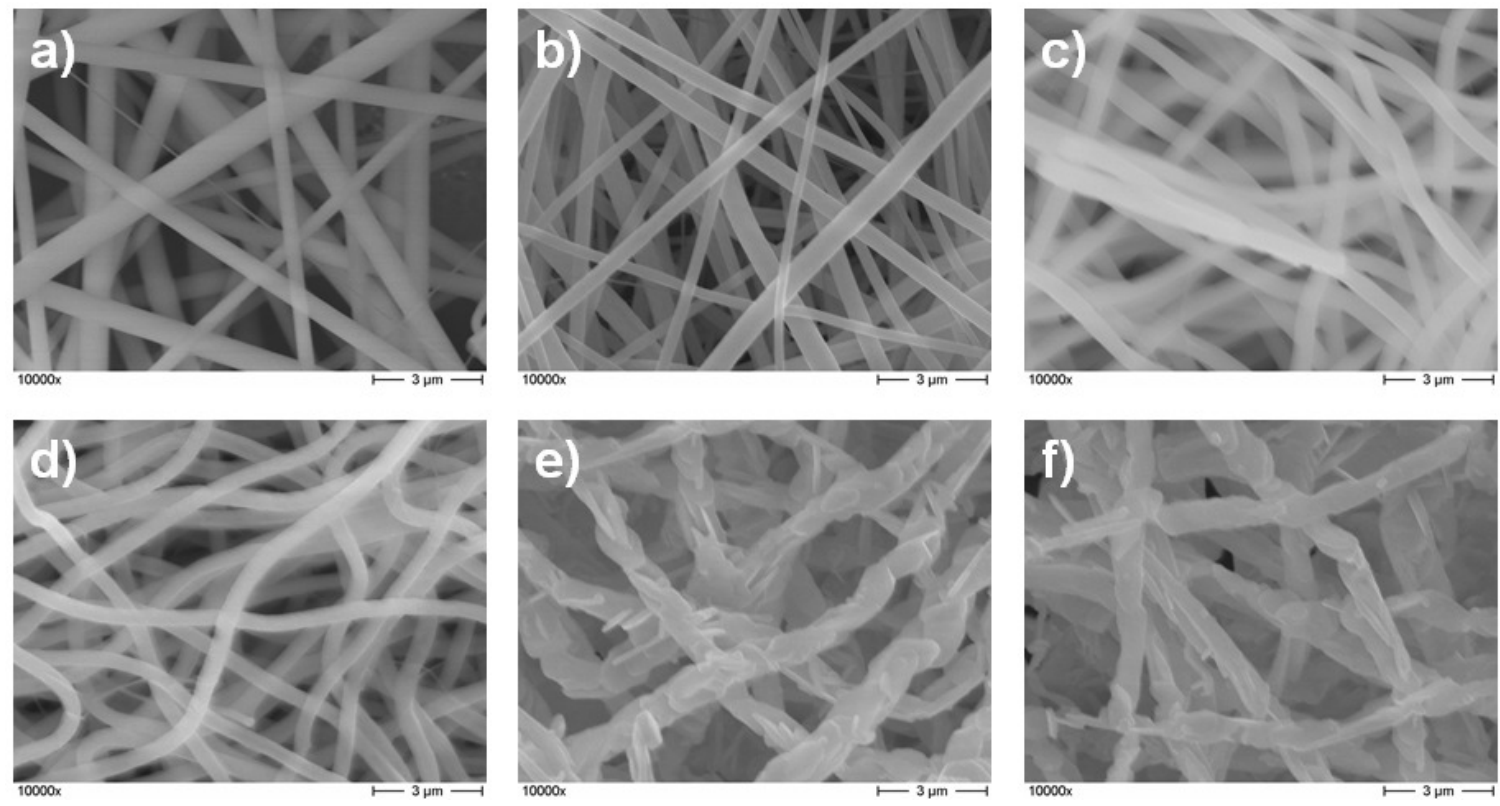

FIG. 2. SEM image of the undoped nanowires (a)-(e) and Pb-doped nanowires (f) at magnification $(10000 \times)$ at different thermal treatment steps. (a) As-prepared, (b) $211{ }^{\circ} \mathrm{C}$, (c) $302{ }^{\circ} \mathrm{C}$, (d) 385 ${ }^{\circ} \mathrm{C}$, and images (e), (f) give the microstructures obtained after a treatment at $800{ }^{\circ} \mathrm{C}$ in air and $500{ }^{\circ} \mathrm{C}$ in $\mathrm{O}_{2}$ environment, respectively.

Figure 3 shows the X-ray diffraction results of the 1112 (a) and the Pb-doped samples (b) via a high-resolution automated RINT2200 X-ray powder diffractometer, using Cu$\mathrm{K}_{\alpha}$ radiation generated at $40 \mathrm{kV}$ and $40 \mathrm{~mA}$. It turns out that with both precursors, the nanowires can obtain the Bi-2212 phase after the thermal treatment. According to the analysis of the peaks, the average grain size of the 1112 sample is about $46.1 \mathrm{~nm}$. However, the 5\% Pb-doped sample presents a worse crystallinity than the 1112 sample, as the peaks from the spectrum have larger FWHM (full width at half maximum) values, and the average grain size is also found to be smaller, i.e., $39.1 \mathrm{~nm}$. This can be attributed to the influence of the lead addition, as the $\mathrm{Pb}$-doping faciliates the formation of the Bi-2212 phase, however, it also induces a lattice distortion.

The nanowires obtained from electrospinning method always have a variation of the diameter. Figure 4 shows the distribution of the wire diameter from both samples. The value has a range from 140 to $580 \mathrm{~nm}$. Table II shows the structure comparison of the 1112 sample and the Pb-doped sample. Generally, the two different types of samples share the similar structure, except that the grain size of the Pb-doped one is smaller. The average 


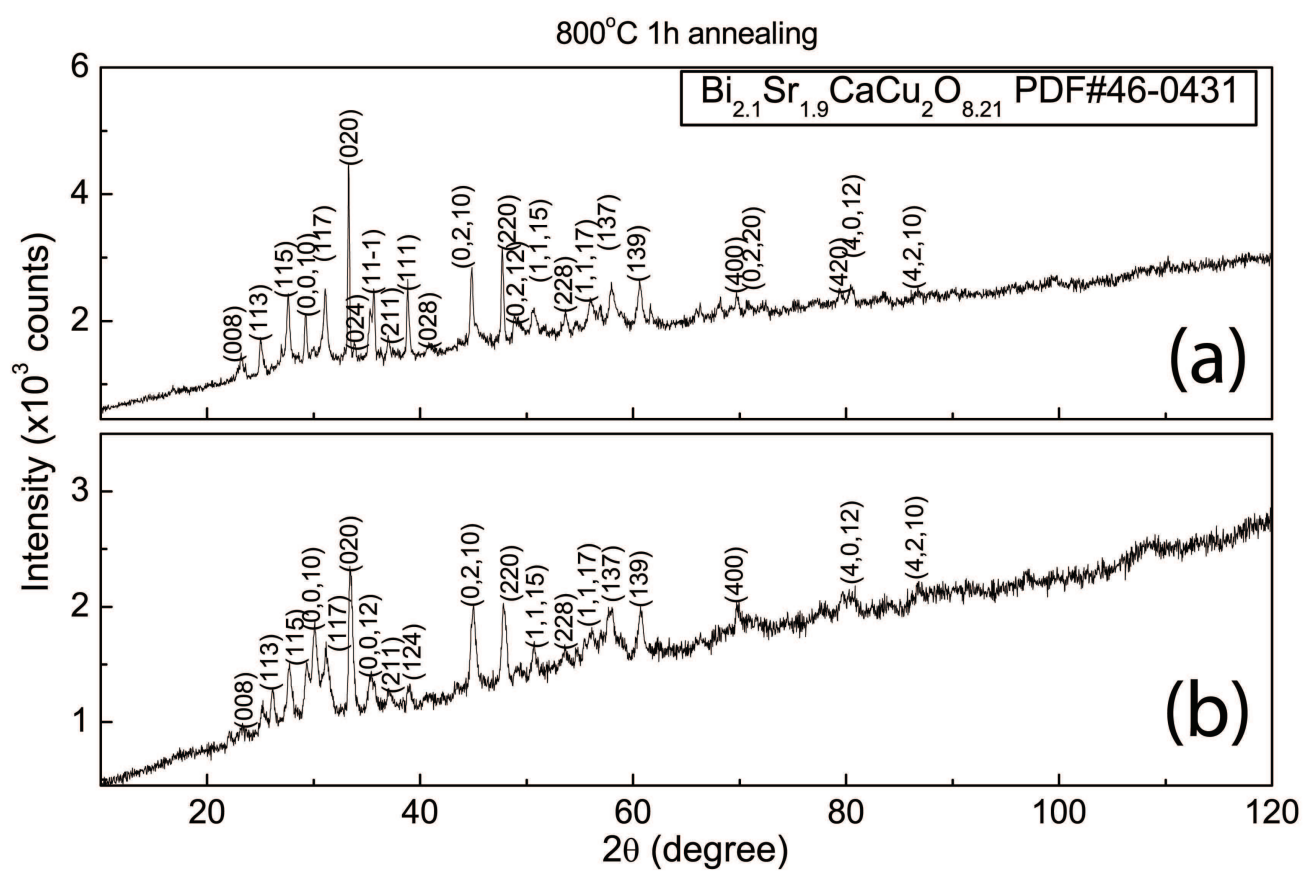

FIG. 3. X-ray diffraction analysis of the nanowires samples after the whole thermal treatment, the phases have been identified as $\mathrm{Bi}_{2.1} \mathrm{Sr}_{1.9} \mathrm{CaCu}_{2} \mathrm{O}_{8.21}$ by the standard PDF card \#46-0431. (a) the 1112 sample, and (b) the $\mathrm{Pb}$-doped sample.

\begin{tabular}{|c|c|c|}
\hline Parameters (averaged) & 1112 & Pb-doped \\
\hline Length $(\mu \mathrm{m})$ & 103.6 & 87.5 \\
\hline Diameter $(\mathrm{nm})$ & 317.6 & 328.3 \\
\hline Grain size $(\mathrm{nm})$ & 46.1 & 39.1 \\
\hline
\end{tabular}

TABLE II. Structure comparison of the 1112 sample and the Pb-doped sample.

wire diameters of both samples are around $320 \mathrm{~nm}$, which is in the range of the London penetration depth ${ }^{18}$.

\section{EXPERIMENTAL PROCEDURE}

\section{A. Magnetic measurement}

The superconductivity of both the 1112 and Pb-doped samples has been investigated for their magnetic behavior via the superconducting quantum interference device (SQUID). Figure 5 shows their magnetization dependence on temperature, in (a) the 1112 sample 


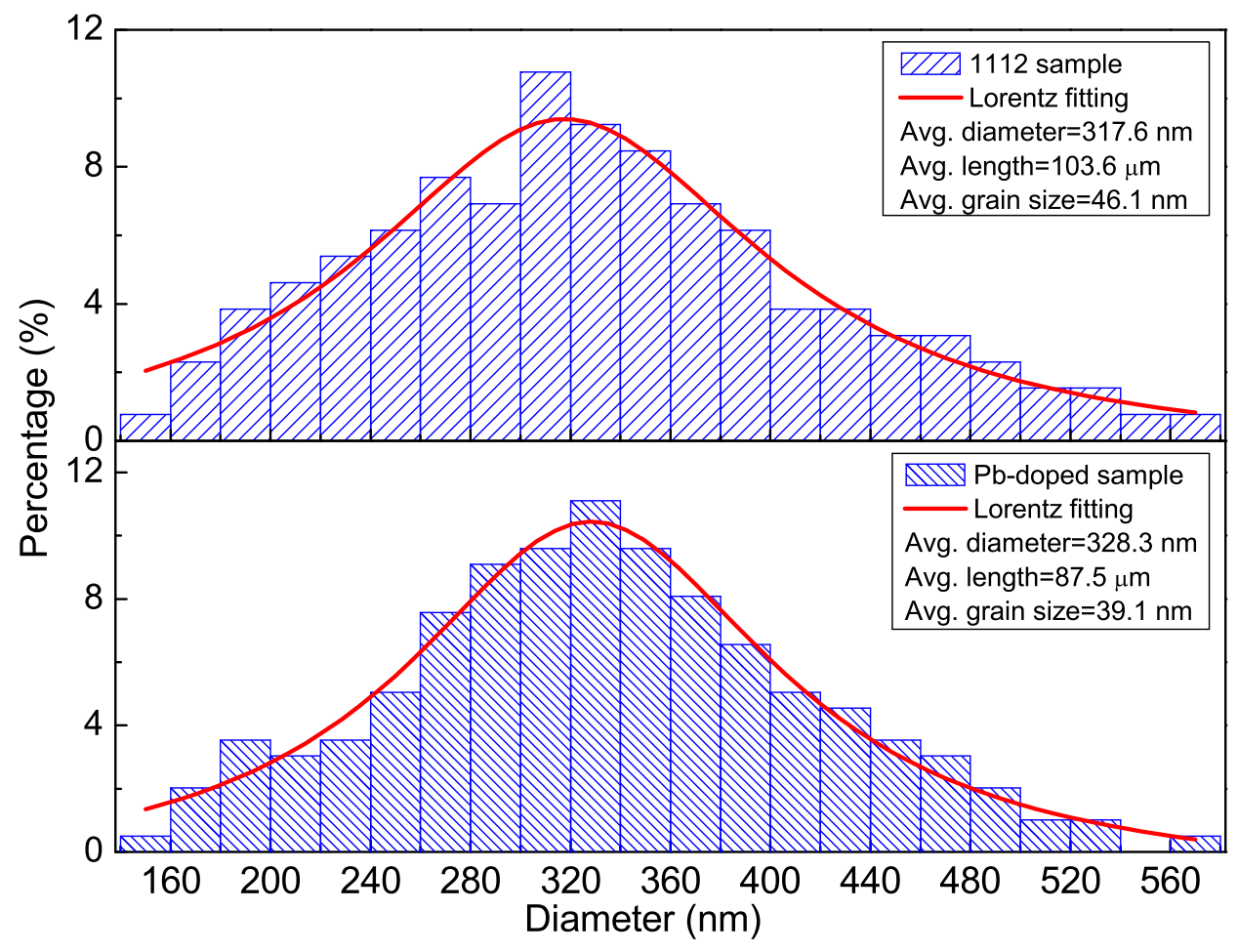

FIG. 4. The diameter statistics of nanoires from the 1112 and the Pb-doped samples. The diameter distributions from both samples have a wide range from $140 \mathrm{~nm}$ to $580 \mathrm{~nm}$. The average diameter of the 1112 sample is about $317.6 \mathrm{~nm}$ and the $\mathrm{Pb}$-doped one is $328.3 \mathrm{~nm}$.

while (b) the Pb-doped sample. As a comparison, the measurements are done in zero field cooling (ZFC) and $1 \mathrm{mT}$ field cooling (FC) modes, during the measurement a detecting field $1 \mathrm{mT}$ is applied. There are two onsets in the $M(T)$ curves. The first one can be defined as the critical temperature $\left(T_{\mathrm{c}}\right)$ at which the samples change from normal state to superconducting state, as the samples become diamagnetic below this temperature. The second onset is the one related to the magnetic irreversibility where the magnetic behavior becomes irreversible below such a temperature ${ }^{19}$. For the 1112 sample, the $T_{\mathrm{c}}$ is obtained around $76 \mathrm{~K}$ while for the $\mathrm{Pb}$-doped sample $T_{\mathrm{c}}$ is located around $84 \mathrm{~K}$, which is close to the value of the bulk materials at $85 \mathrm{~K}$. According to the result of the FC curves, they start to deviate the ZFC curve at around $70.2 \mathrm{~K}$ and $62.7 \mathrm{~K}$ in the 1112 sample and the $\mathrm{Pb}$ doped sample respectively, thus it can be estimated that the samples show the field irreversiblity at certain temperatures. For the nanowires with their diameter being comparable to the penetration depth, the relatively lower $T_{\mathrm{c}}$ from the 1112 sample can be attributed to thermal and quantum fluctuations induced by the decreasing size of the sample ${ }^{20,21}$. On the other 
hand, with the $\mathrm{Pb}$-doping, the sample shows a higher $T_{\mathrm{c}}$. The influence of $\mathrm{Pb}$-doping on the superconducting properties of Bi-2212 has been investigated for a decade in bulk and thin film materials. It is well known that the additional $\mathrm{Pb}$ serves as a substitution of the bismuth in the lattice structure. Thus, it can increase the volume fraction of the superconducting phase $^{22}$ and reduce the electronic anisotropy of Bi- $2212^{23}$. A certain amount of Pb-doping could introduce an enhancement of the superconductivity. However, with increasing doping level, the lattice distortion due to the $\mathrm{Pb}$ substitution will also produce a suppression of the superconductivity ${ }^{24}$.

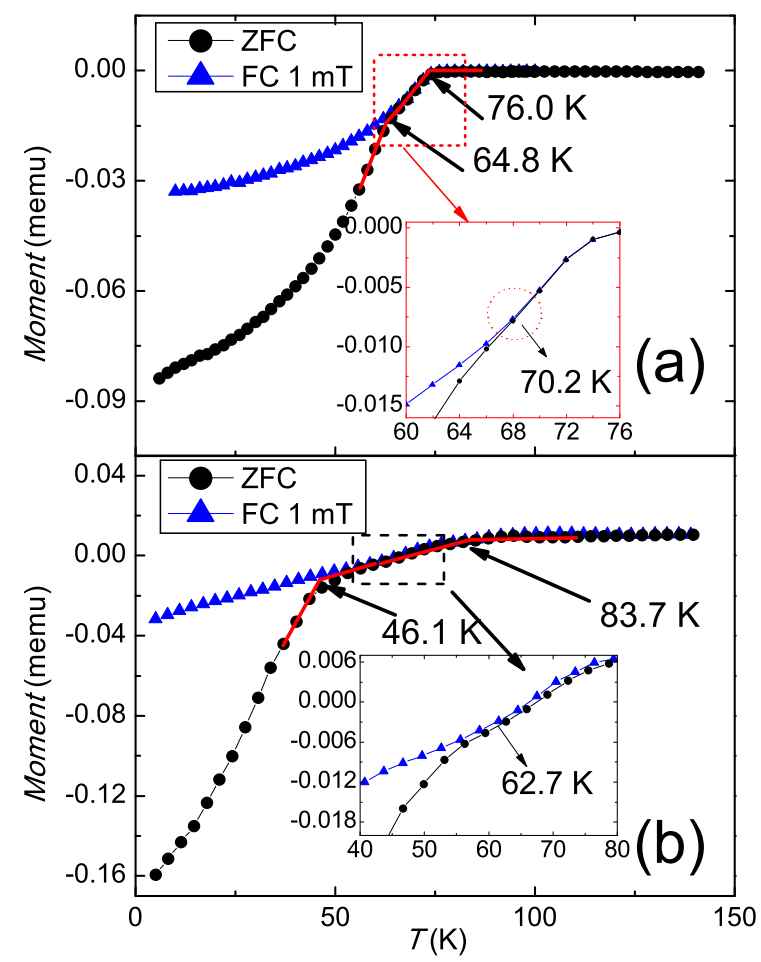

FIG. 5. Magnetization dependence on temperature of the nanowires. The onsets at each curve can be related to the $T_{\mathrm{c}}$ and the irreversibility temperature of the sample. (a) shows the 1112 sample and (b) the Pb-doped sample.

Figure 6 gives a comparison of the magnetization loops of the 1112 sample (a) and Pbdoped sample (b) in fields up to $\pm 7 \mathrm{~T}$ in the temperature range from $5 \mathrm{~K}$ to $70 \mathrm{~K}$. Starting from $5 \mathrm{~K}$, all the loops get fully asymmetric. The so-called fishtail effect ${ }^{25}$ in the magnetization loops can be seen in form of a minimum of the magnetic moment close to the full penetration field [see the upper inset to 5 (b)], and double sharp peaks occur close to zero field. Furthermore, the magnetization loops are asymmetric at the axis $M=0$. Compared 


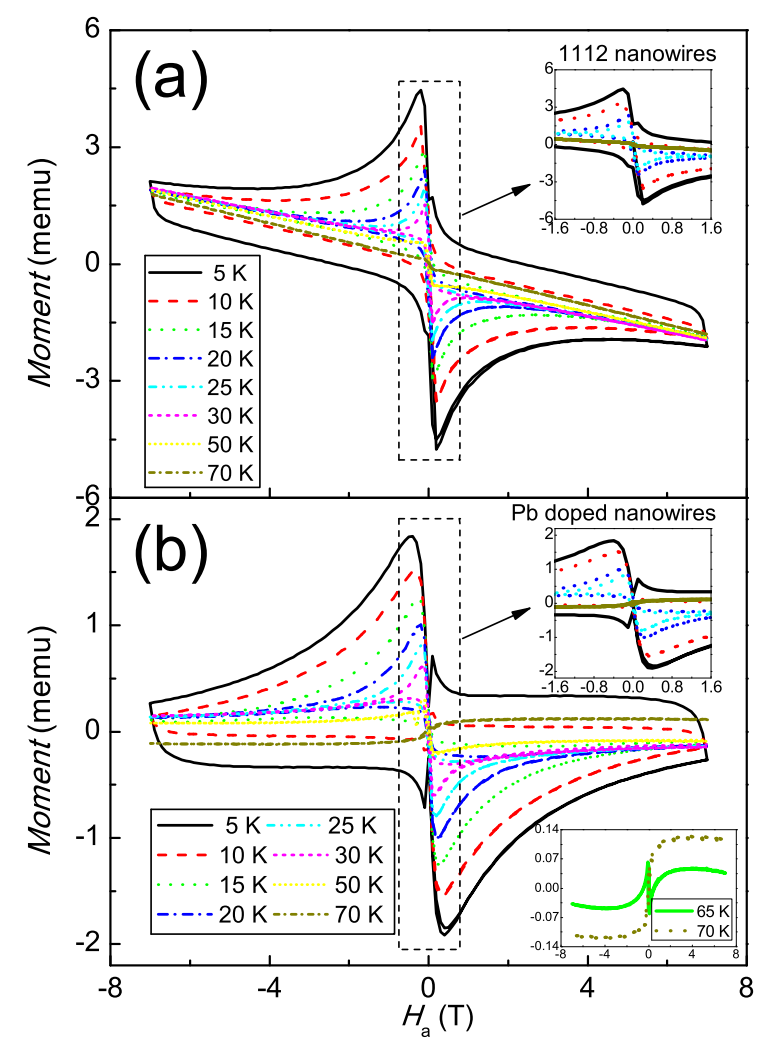

FIG. 6. The comparison of the $M(H)$ loops of the samples ranging from $5 \mathrm{~K}$ to $70 \mathrm{~K}$. (a) gives the data of the 1112 sample, and the inset presents the low-field part in detail. (b) shows the $M(H$ of the Pb-doped sample. The upper inset presents details for low fields, and the lower inset shows the magnetization loops at $65 \mathrm{~K}$ and $70 \mathrm{~K}$ in detail.

to the typical symmetric loop of bulk cuprate superconductors ${ }^{26}$, the loops from both samples show a completely different behavior which reveals the polycrystalline character of the granular nanowires. In the case of the 1112 sample, the irreversible superconducting loop maintains up to $70 \mathrm{~K}$, corresponding to the result obtained from the $M(T)$ curve where $T_{\mathrm{c}}$ is about $76 \mathrm{~K}$. The diamagnetic background superposed on the loops can be attributed to the excess of $\mathrm{Cu}$ from the preparation, which forms the $\mathrm{CuO}$ impurity.

In the case of the $\mathrm{Pb}$-doped sample, the irreversible superconducting loop vanishes above $65 \mathrm{~K}$, and a reversible paramagnetic curve is observed. This demonstrates that above this temperature the irreversibility due to the flux pinning is gone, and no vortices are pinned in the sample. Such result has a good agreement with the irreversibiity starts at around 63 $\mathrm{K}$ from the $M(T)$ curve. We can conclude here that the pinning landscape of the $\mathrm{Pb}$-doped sample is worse as compared to the 1112 sample, especially at high temperatures. 


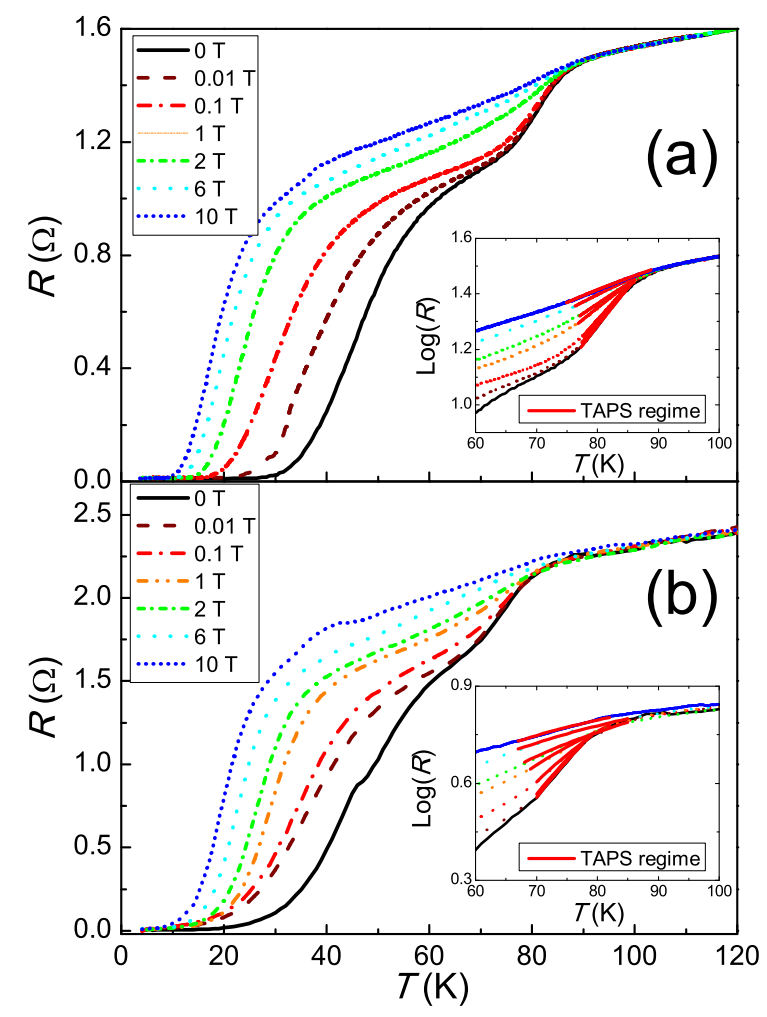

FIG. 7. Comparison of the resistance-temperature behavior of the samples as a function of temperature ranging from $4 \mathrm{~K}$ to $120 \mathrm{~K}$, with an applied current of $50 \mu \mathrm{A}$. (a) gives the data of the 1112 sample and (b) the data of the Pb-doped sample.

\section{B. Electric properites}

In order to clarify whether the $\mathrm{Pb}$-doping has a positive or negative influence to the superconductivity of the sample, four-probe measurement is applied to investigate the resistance dependence on temperature. Before the connection to the electrodes, the samples have been coated with $100 \mathrm{~nm}$ gold as to enhance the contact quality.

Figure 7 shows the results of both samples from $4 \mathrm{~K}$ to $120 \mathrm{~K}$ in the field range from 0 T to $10 \mathrm{~T}$ [(a) for 1112 sample and (b) for Pb-doped sample]. The two step behavior of the resistance drop occurs in both samples. The first drop of resistance stems from the sample phase transition from the normal state to the superconducting state. The values of the normal state resistance $R_{N}{ }^{28}$ can be estimated as $1.59 \Omega$ at $115.7 \mathrm{~K}$ and $2.25 \Omega$ at 87.5 $\mathrm{K}$ from the $0 \mathrm{~T}$ curves of the 1112 sample and the $\mathrm{Pb}$ doped sample respectively. However, it is difficult to measure the crosssection area of the samples, so the comparison of $\rho_{N}$ of 


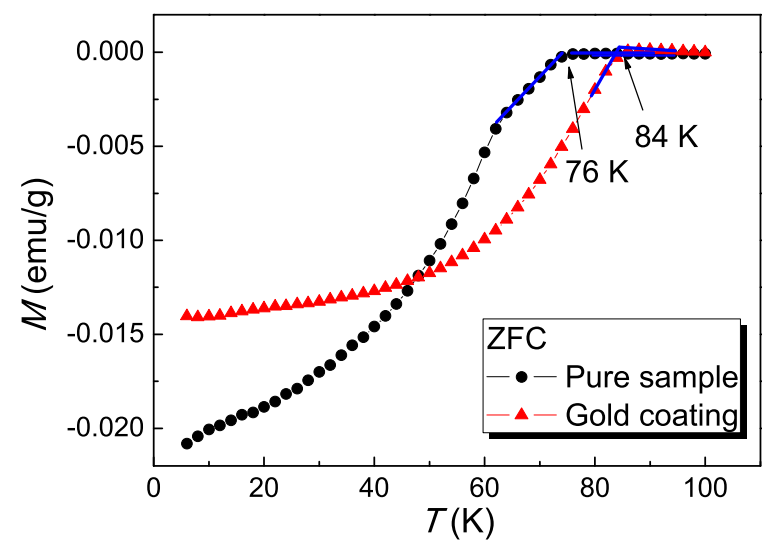

FIG. 8. $M(T)$ measurements before and after the gold coating

the samples is not available. The non-zero resistance broadening of the curves around $T_{\mathrm{c}}$ shows an exponential relationship with the inverse of the temperature. Such regimes can be fitted by the mathematic expression $\exp (-\Delta F / k T$ ) (see the insets to Fig. 7). $\Delta F$ is the energy required to locally suppress superconductivity. This indicates that the resistances are resulting from the thermal activated phase slip (TAPS ${ }^{27}$. The residual resistance at the second step is the one due to the intergrain weak links inside the nanowires ${ }^{29}$. With increasing field, the regime of the first step becomes even broader due to the enhanced phase slip rate with field ${ }^{30}$. Such effect is more sensitive than the variation of $R_{N}$ in field, thus the two steps get closer at higher field. At $10 \mathrm{~T}$ applied field, the steps merge to one.

In the case of the 1112 sample, the onset of superconductivity is about $85 \mathrm{~K}$, which means that the value of critical temperature is higher than the one from the $M(T)$ curve, $76 \mathrm{~K}$. The $9 \mathrm{~K}$ enhancement can be attributed to the influence of the coating gold layer, which has been proven by the comparison of the $M(T)$ curves with and without gold coating (Figure ??). To the Pb-doped sample, the $0 \mathrm{~T}$ curve shows a $T_{\mathrm{c}}$ around $78 \mathrm{~K}$. Considering the same thickness of gold layer which contributes a similar increase to the $T_{\mathrm{c}}$, we can estimate that the $T_{\mathrm{c}}$ of the $\mathrm{Pb}$-doped sample is about $68 \mathrm{~K}$, which is much lower than the one obtained from the $M(T)$ curve.

The superconductivity increasement from the gold is opposite to the proximity effect that metal layer decreases the $T_{\mathrm{c}}$ of the superconductor. One of the possible explanation is that the gold layer introduces the intergrain Josephson coupling to the sample, leading to a higher $T_{\mathrm{c}}$ ? . 


\section{CONCLUSION}

The Bi-2212 nanowire networks have been fabricated by electrospinning method and the consequent thermal treatment. In order to obtain the superconducting phase, the excess of copper, calcium salts and the Pb-doping (as the substitution of bismuth) are applied as two treatments to the precursors. The samples from both precursors show a similar polycrystalline nanowire structure with the wire diameter comparable to the penetration depth. According to the XRD result, the 1112 sample is better crystallized than the $\mathrm{Pb}-$ doped sample. The magnetic measurements reveal clear differences between the two samples. From the $M(T)$ measurement, the Pb-doped sample has a higher value of $T_{\mathrm{c}}$ than the 1112 sample, however, the $M(H)$ measurement shows the opposite. In the $R(\mathrm{~T})$ measurement, a two-step drop of the resistance is observed in both samples. The nonzero resistance from these two steps can be explained by the influence of TAPS and intergrain weak links inside the nanowires. The gold coating on the sample surface successfully solves the Schottky contact problem between the sample and the electrodes, giving a positive influence to the superconductivity. The $T_{\mathrm{c}}$ results from the $R(\mathrm{~T})$ show a good agreement with the ones obtained from the $M(H)$ measurements. As a conclusion of the present measurements, both excess of $\mathrm{Cu}-, \mathrm{Ca}$ - and $\mathrm{Pb}$-doping can help to form the $\mathrm{Bi}-2212$ phase. The sample synthesized from the former method presents a better crystallinity, relative higher $T_{\mathrm{c}}$ than the latter one. It indicates that to the electrospinnng polycrystalline nanowire, the influence of lattice distortion from $\mathrm{Pb}$-doping is dominant.

\section{ACKNOWLEDGMENTS}

We thank Prof. V. Presser (INM Saarbrücken and Saarland University) for the possibility to use the electrospinning apparatus and Dr. J. S. Atchinson (INM Saarbrücken) and J. Schmauch (Saarland University, group Prof. Birringer) for technical assistance. This work is supported by Volkswagen Foundation. The collaboration UdS-Nancy was supported by an EU-INTERREG IVa project "Greater Region Magnetism Network (GRMN)". 


\section{REFERENCES}

${ }^{1}$ A. Bezryadin, C. N. Lau, and M. Tinkham, "Quantum suppression of superconductivity in ultrathin nanowires", Nature 404, 971 (2000).

${ }^{2}$ S. H. Lai, Y. C. Hsu, and M. D. Lan, "Synthesis of $\mathrm{Bi}_{2} \mathrm{Sr}_{2} \mathrm{CaCu}_{2} \mathrm{O}_{y}$ nanowire and its superconductivity", Solid State Commun. 148, 452 (2008).

${ }^{3}$ L. Zhang, L. You, D. Liu, W. Zhang, L. Zhang, X. Liu, J. Wu, Y. He, C. Lv, Z. Wang, and X. Xie, "Characterization of superconducting nanowire single-photon detector with artificial constrictions", AIP Advances 4, 067114 (2014).

${ }^{4}$ A. Korneev, P. Kouminov, V. Matvienko, G. Chulkova, K. Smirnov, B. Voronov, G. N. Goltsman, M. Currie, W. Lo, K. Wilsher, J. Zhang, W. Slysz, A. Pearlman, A. Verevkin, and R. Sobolewski, "Sensitivity and gigahertz counting performance of NbN superconducting single-photon detectors", Appl. Phys. Lett. 84, 5338 (2004).

${ }^{5}$ C. N. Lau, N. Markovic, M. Bockrath, A. Bezryadin, and M. Tinkham, "Quantum phase slips in superconducting nanowires", Phys. Rev. Lett. 87, 217003 (2001).

${ }^{6}$ D. S. Golubev, and A. D. Zaikin, "Thermally activated phase slips in superconducting nanowires", Phys. Rev. Lett. 78, 1 (2008).

${ }^{7}$ D. S. Golubev, and A. D. Zaikin, "Quantum tunneling of the order parameter in superconducting nanowires", Phys. Rev. B 64, 014504 (2001).

${ }^{8}$ I. Sochnikov, A. Shaulov, Y. Yeshurun, G. Logvenov, and I. Božović, "Large oscillations of the magnetoresistance in nanopatterned high-temperature superconducting films", Nature Nanotechnol. 5, 516 (2010).

${ }^{9}$ E. Bar, D. Levi, G. Koren, A. Shaulov, and Y. Yeshurun, "Transport properties of ultrathin granular $\mathrm{YBa}_{2} \mathrm{Cu}_{3} \mathrm{O}_{7-\delta}$ nanobridges", Physica C 506, 160 (2014).

${ }^{10}$ J. Fransaer, J. R. Roos, L. Delaey, O. Van Der Biest, O. Arkens, and J. P. Celis, "Sol-gel preparation of high- $T_{\mathrm{c}}$ Bi-Ca-Sr-Cu-O and Y-Ba-Ca-O superconductors", J. Appl. Phys. 65, $3277(1989)$

${ }^{11}$ D. Li, J. T. McCann, Y. Xia, and M. Marquez, "Electrospinning: a simple and versatile technique for producing ceramic nanofibers and nanotubes", J. Am. Ceram. Soc. 89, 1861 (2006).

${ }^{12}$ J. I. Gorina, G. A. Kaljujnaia, V. I. Ktitorov, V. P. Martovitsky, V. V. Rodin, V. A. Stepanov, A. A. Tsvetkov, and S. I. Vedeneev, "Superconducting and structrual properties 
of homogeneous BiSrCaCuO (2212) single crystals prepared by solution growth", Solid State Commun. 85, 695 (1993).

${ }^{13}$ I. Chong, Z. Hiroi, M. Izumi, J. Shimoyama, Y. Nakayama, K. Kishio, T. Terashima, Y. Bando, and M. Takano, "High critical-current density in the heavily Pb-doped $\mathrm{Bi}_{2} \mathrm{Sr}_{2} \mathrm{CaCu}_{2} \mathrm{O}_{8+\delta}$ superconductor: Generation of efficient pinning centers", Science 276, 770 (1997).

${ }^{14}$ J. Shimoyama, Y. Nakayama, K. Kitazawa, K. Kishio, Z. Hiroi, I. Chong, and M. Takano, "Strong flux pinning up to liquid nitrogen temperature discovered in heavily $\mathrm{Pb}$-doped and oxygen controlled Bi2212 single crystals", Physica C 281, 69 (1997).

${ }^{15}$ J. S. Atchinson, M. Zeiger, A. Tolosa, L. M. Funke, N. Jäckel, and V. Presser, "Electrospinning of ultrafine metal oxide/carbon and metal carbide/carbon nanocomposite fibers", RSC Adv. 5, 35683 (2005).

${ }^{16}$ J. M. Li, X. L. Zeng, A. D. Mo, and Z. A. Xu, "Fabrication of cuprate superconducting $\mathrm{La}_{1.85} \mathrm{Sr}_{0.15} \mathrm{CuO}_{4}$ nanofibers by electrospinning and subsequent calcination in oxygen", CrystEngComm. 13, 6964 (2011).

${ }^{17}$ X. L. Zeng, M. R. Koblischka, and U. Hartmann, "Synthesis and characterization of electrospun superconducting ( $\mathrm{La}, \mathrm{Sr}) \mathrm{CuO}_{4}$ nanowires and nanoribbons", Mat. Res. Express 2, $095022(2015)$.

${ }^{18}$ R. Prozorov, R. W. Giannetta, A. Carrington, P. Fournier, R. L. Greene, P. Guptasarma, D. G. Hinks, and A. R. Banks, "Measurements of the absolute value of the penetration depth in high- $T_{\mathrm{c}}$ superconductors using a low- $T_{\mathrm{c}}$ superconductive coating", Appl. Phys. Lett. 77, 4202 (2000).

${ }^{19}$ M. R. Koblischka, X. L. Zeng, T. Karwoth, T. Hauet, and U. Hartmann, "Magnetic properties of electrospun non-woven superconducting fabrics", AIP Advances 6, 035115 (2016).

${ }^{20}$ T. Aref, A. Levchenko, V. Vakaryuk, and A. Bezryadin, "Quantitative analysis of quantum phase slips in superconducting $\mathrm{Mo}_{76} \mathrm{Ge}_{24}$ nanowires revealed by switching-current statistics", Phys. Rev. B 86, 024507 (2012).

${ }^{21}$ M. Tinkham, C. N. Lau, and N. Markovic, "Resistance induced by quantum phase-slips in superconducting nanowires", Physica E 18, 308 (2003).

${ }^{22}$ N. Hudakova, V. Plechacek, P. Dordor, K. Flachbart, K. Knizek, J. Kovac, and M. Reiffers, "Influence of $\mathrm{Pb}$ concentration on microstructural and superconducting properties of 
BSCCO superconductors", Supercond. Sci. Technol. 8, 324 (1995).

${ }^{23}$ L. Winkeler, S. Sadewasser, B. Beschoten, H. Frank, F. Nouvertné, and G. Güntherodt, "Interlayer coupling in Pb-substituted $\mathrm{Bi}_{2} \mathrm{Sr}_{2} \mathrm{CaCu}_{2} \mathrm{O}_{8}$ single crystals", Physica C 265, 194 (1996).

${ }^{24}$ A. Sotelo, Sh. Rasekha, G. Constantinescu, H. Amaveda, M. A. Torres, M. A. Madre, and J. C. Diez, "Effect of $\mathrm{Pb}$ doping on the electrical properties of textured Bi-2212 superconductors", J. Eur. Ceram. Soc. 34, 2977 (2014).

${ }^{25}$ M. Werner, F. M. Sauerzopf, H. W. Weber, and A. Wisniewski, "Fishtail effect in the magnetization of superconducting $\mathrm{RBa}_{2} \mathrm{Cu}_{3} \mathrm{O}_{7-\delta}(\mathrm{R}=\mathrm{Y}, \mathrm{Nd}, \mathrm{Yb})$ and $\mathrm{Y}_{2} \mathrm{Ba}_{4} \mathrm{Cu}_{8} \mathrm{O}_{16}$ single crystals", Phys. Rev. B 61, 14795 (2000).

${ }^{26}$ O. Kizilaslan, G. Kirat, and M.A. Aksan, "Magnetic relaxation behavior in the $\mathrm{Bi}_{2} \mathrm{Sr}_{2} \mathrm{Ca}_{2} \mathrm{Cu}_{3-x} \mathrm{Mo}_{x} \mathrm{O}_{10+\delta}$ system fabricated by glass-ceramic technique", J. Magn. Magn. Mater. 384, 186 (2015).

${ }^{27} \mathrm{~K} . \mathrm{Xu}$, and J. R. Heath, "Long, highly-ordered high-temperature superconductor nanowire arrays", Nano Lett. 8, 3845 (2008).

${ }^{28}$ H. M. Jaeger, D. B. Haviland, B. G. Orr, and A. M. Goldman, "Onset of superconductivity in ultrathin granular metal films", Phys. Rev. B 40, 182 (1996).

${ }^{29}$ A. T. Bollinger, A. Rogachev M. Remeika, and A. Bezryadin, "Effect of morphology on the superconductor-insulator transition in 1-dimensional nanowires" Phys. Rev. B 69, 180503 (2004).

${ }^{30}$ M. Tinkham, "Resistive transition of high-temperature superconductors", Phys. Rev. Lett. 61, 1658 (1988).

${ }^{31}$ O. Yuli, I. Asulin, O. Millo, and D. Orgad, "Enhancement of the superconducting transition temperature of $\mathrm{La}_{2-x} \mathrm{Sr}_{x} \mathrm{CuO}_{4}$ bilayers: Role of pairing and phase stiffness", Phys. Rev. Lett. 101, 4 (2008). 